\title{
A Phytosociological of Plant Communities and Biodiversity in the East-South of Idna Village-Hebron of Palestine
}

\author{
Jehad M. H. Ighbareyeh ${ }^{1,2^{*}}$, Eusebio Cano Carmona ${ }^{1}$ \\ ${ }^{1}$ Faculty of Experimental Sciences, Department of Animal and Plant Biology and Ecology, University of Jaen, \\ Jaen, Spain \\ ${ }^{2}$ Faculty of Agriculture, Al-Quds Open University, Hebron, Palestine \\ Email: ^jehadighbareyeh@hotmail.com
}

How to cite this paper: Ighbareyeh J.M.H., and Cano Carmona, E. (2018) A Phytosociological of Plant Communities and Biodiversity in the East-South of Idna Village-Hebron of Palestine. International Journal of Geosciences, 9, 44-58. https://doi.org/10.4236/ijg.2018.91003

Received: November 29, 2017

Accepted: January 15, 2018

Published: January 18, 2018

Copyright $\odot 2018$ by authors and Scientific Research Publishing Inc. This work is licensed under the Creative Commons Attribution International License (CC BY 4.0).

http://creativecommons.org/licenses/by/4.0/

\section{(c) (i) Open Access}

\begin{abstract}
This paper presents a floristic and vegetation study of the territories of the Idna-Hebron of Palestine, corresponding to one area with three different names as Abu Salman Forest Reserve, Khallet Osman mountains, and Hamra Aslimi. These sites are very important at a local level of the flora with a high endemic rate. The floristic analysis revealed the existence of 48 species, of which $10(20.83 \%)$ are endemic endemic. Seventeen plots of vegetation distributed in one area dominated and have been analyzed in this study. Methodology, the phytosociological approach is based on the Braun-Blanquet method. The results revealed three different kinds of forests, one dominated by the endemic Pino halepensis-Quercetum lookii, which is peculiar to the inframediterranean dry-thermomediterranean environments, with the terra rossa and brown rendzinas territory. The second type of forest is dominated by the Pistacio palaestinae-Ceratonietum silique, which is growing in the dry-subhumid ombrotype and the terra rossa and brown rendzinas territory. The third group of the forest of Khallet Osman and Hamra Aslimi dominated by Quercus lookii-Tamaricetum palestineae. Finally, in this study there are three associations and tow alliances are proposed as new Syntaxa based on statistical and phytosociological analyses in the study area.
\end{abstract}

\section{Keywords}

Palestine, Phytosociology, Idna, Abu Salman Forest Reserve, Vegetation, Biodiversity, Flora

\section{Introduction}

Palestine has a wide range of agro-ecological concerns, particular geographic 
location, with a series of environmental, a unique biodiversity and a large variety of plants [1] [2] [3], it has a Mediterranean climate, characterized by mild rainy winters and hot dry summers, with a tropical-subtropical tendency in the far south, and rainfall decreases from north to south, while the temperature increases [2]. Climate and bioclimate were played an important role in influence on plant communities, plant production, and biological resources [1]-[20].

In Palestine, about 2780 plant taxa were recorded as native, which of 159 [19], 162 taxa were recorded as endemics [20] [21]. Moreover, it is found that about 2750 species of plants including 138 families were estimated for Palestinian flora [22], [23], while in the other study the flora of Palestine includes 149 endemic species [24], (6\% of the total flora), which of $43 \%$ are found to be common, $27.5 \%$ are rare and $25.6 \%$ are very rare. The recent study there recorded more than 1881 species of plant, which of 53 are endemic rare species in the fourth areas of Palestine as Hebron, Ramallah, Jericho and Jenin [19], and we described eight new plant associations, of which two have a dry-sub-humid ombrotype (Pistacio palaestinae-Quercetum lokii; Cerasus microcarpae-Quercetum ithaburensis) in the area of Idna, Hebron of Palestine [3].

The aim of this paper is to provide a botanical study of the flora and vegetation growing in the Idna-Hebron of Palestine.

\section{Materials and Methods}

In our study and survey consists of up to 17 vegetation grids distributed over one area with three different names as (Abu Salman Forest Reserve, Khallet Osman and Aslimi) (Figure 1). The sampling area ranged from 400 to $430 \mathrm{du}-$ nums, according to the vegetation unit concerned, forest and very little scrubland (trees only). Nevertheless, for each one, we provide the records of altitude, slope, area, cover rate, orientation and number of species. In our phytosociological

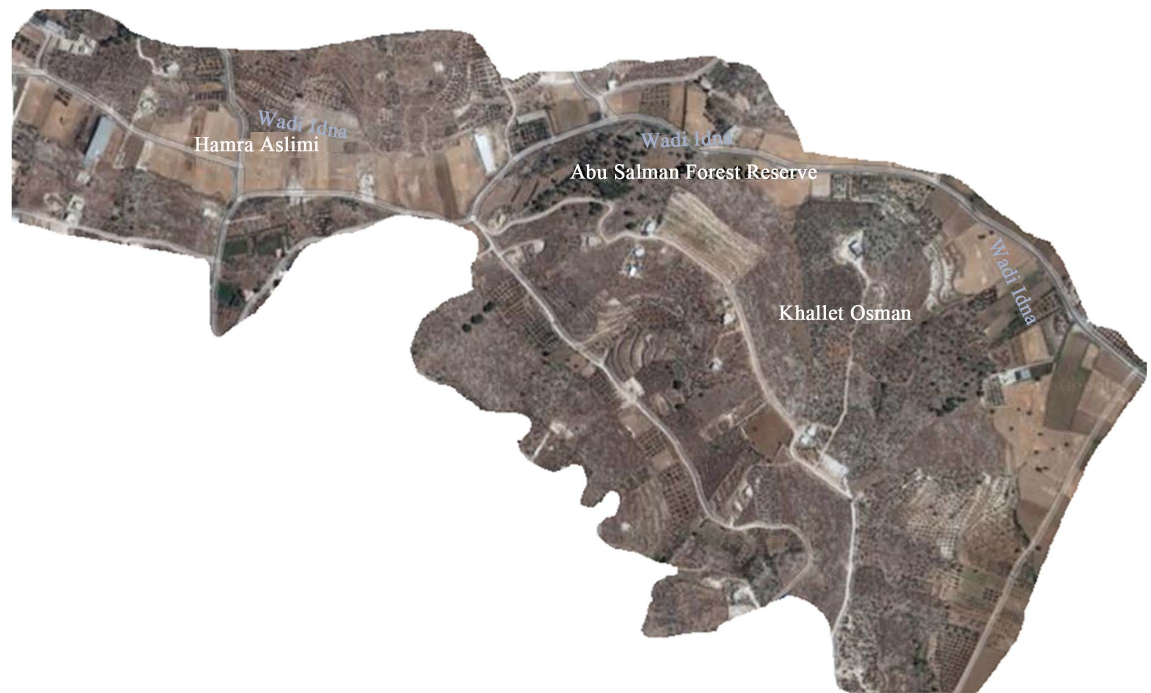

Figure 1. Represent the study area in Idna village by satellite. 
approach is fundamentally based on the Braun-Blanquet (1979) [25] method, we have taken into account the advances in the method pointed out by Pott (2011) [26] and Biondi (2011) [27].

However, we made a comprehensive floristic study of these 17 grids, singled out the respective endemic species and identified the floristic differences in the territories involved. The phytosociological indexes are converted to the Van der Maarel (1979) [28] scale for a proper statistical analysis, these data were used to create an Excel table with 48 rows (plants) and 17 columns (grids). Moreover, from this table we generated a Euclidean Distance Matrix to measure the distance, with the relevant data on community plant physiognomy, average height of dominant species and abundance dominance index, we created a table with the dominant species of each of the 17 grids. We took values over $6 \mathrm{~m}$ as the average forest height, dominant species with average height values ranging from 3.5 to $7 \mathrm{~m}$ could be described to forest as well as to scrubland environments as Cerasus microcarpa Boiss, Rhamnus palaestinus Boiss. The bioclimatic analysis was carried out based on climatic data provided by Palestine Meteorological Department and according to the Rivas-Martinez bioclimatic system (RivasMartinez et al. 2011) [29], which main aim was to study the relationship between the climate and the living species and their communities on the land in the world.

\section{Study Area}

Idna (Idhna) is Palestinian village located to the north-west of the city of Hebron (13 kilometers west of Hebron) and in the southern West Bank, one of the territories occupied in 1967 and is located on the Green Line, an area now estimated at 17,000 hectares after it was 37,000 hectares in 1948, elevated about $450-500$ $\mathrm{m}$ above sea level, between longitudes $34^{\circ} 06^{\prime}$ east and latitudes $31^{\circ} 33^{\prime}$ north. In the fact, we took one area is located at the east-south of Idna village that's have three different names as Abu Salman Forest Reserve (elevation 450 - $545 \mathrm{~m}$ ), Khallet Osman (elevation 440 - $540 \mathrm{~m}$ ) and Hamra Aslimi (elevation 420 - 445 $\mathrm{m}$ ) (Figure 1 and Figure 2).

\section{Results and Discussion}

In our study, the clustering analysis (Euclidean distance) applied to our data matrix of 48 rows (plants) and 17 columns yielded three clearly separated groups at the level of the truncation of (0.93). Group I (P1, P2, P5, P9, P11, P12, P14 and P16), Group II (P3, P4, P7, P8, P13 and P15) show the relives collected in Abu Salman Forest Reserve. Group III (P6, P10 and P17) gathers the relives corresponding to the forests of the Khallet Osman and Hamra Aslimi mountains (Figure 3). Both groups include plots located in the east-south of the Idna village and near of Suba.

However, when we applied of a principal component analysis (PCA) and covariance matrix obtained positive results, thereby confirming the existence of the 


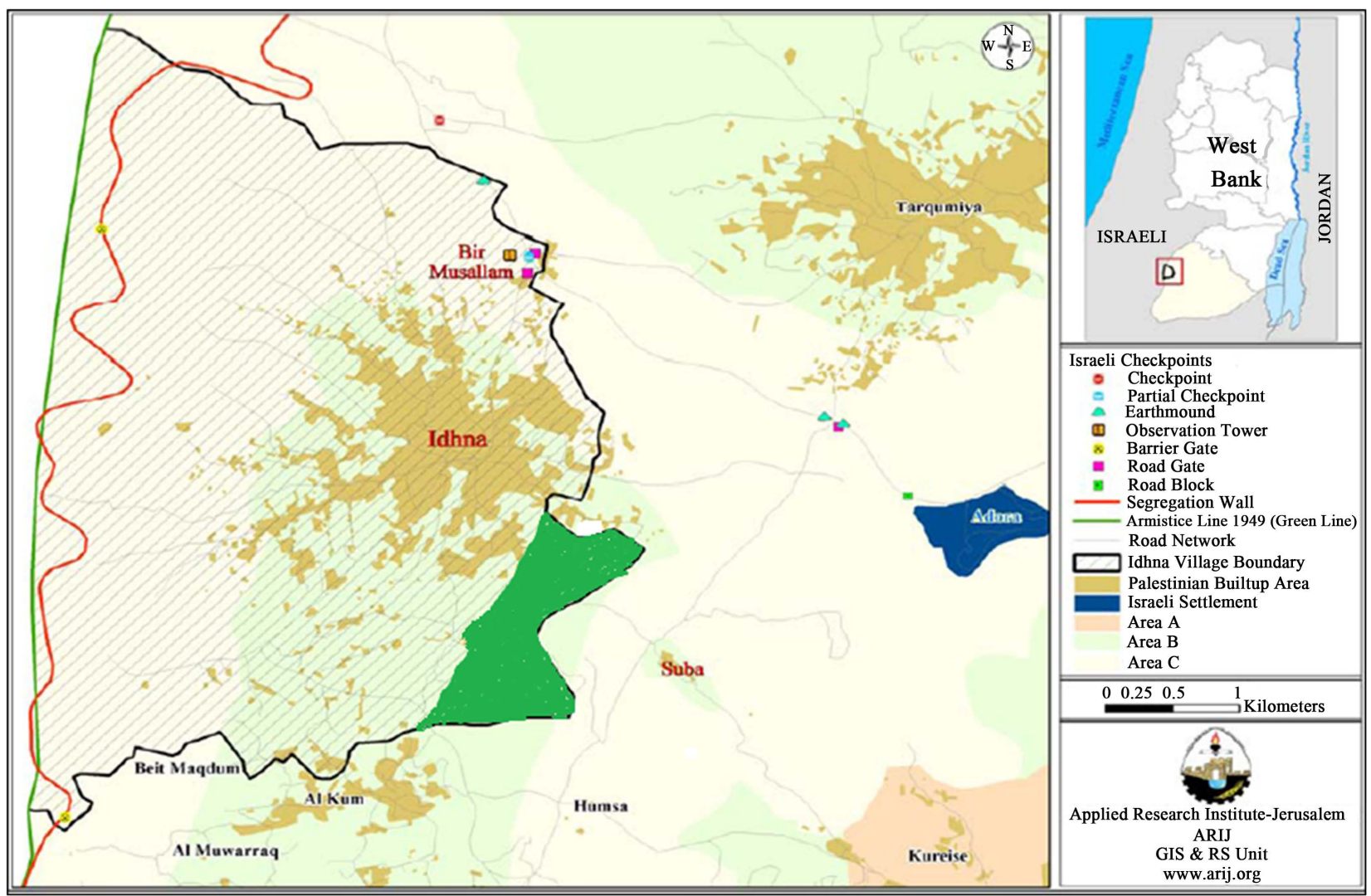

Figure 2. Idna location and green color represent a study area in the map.

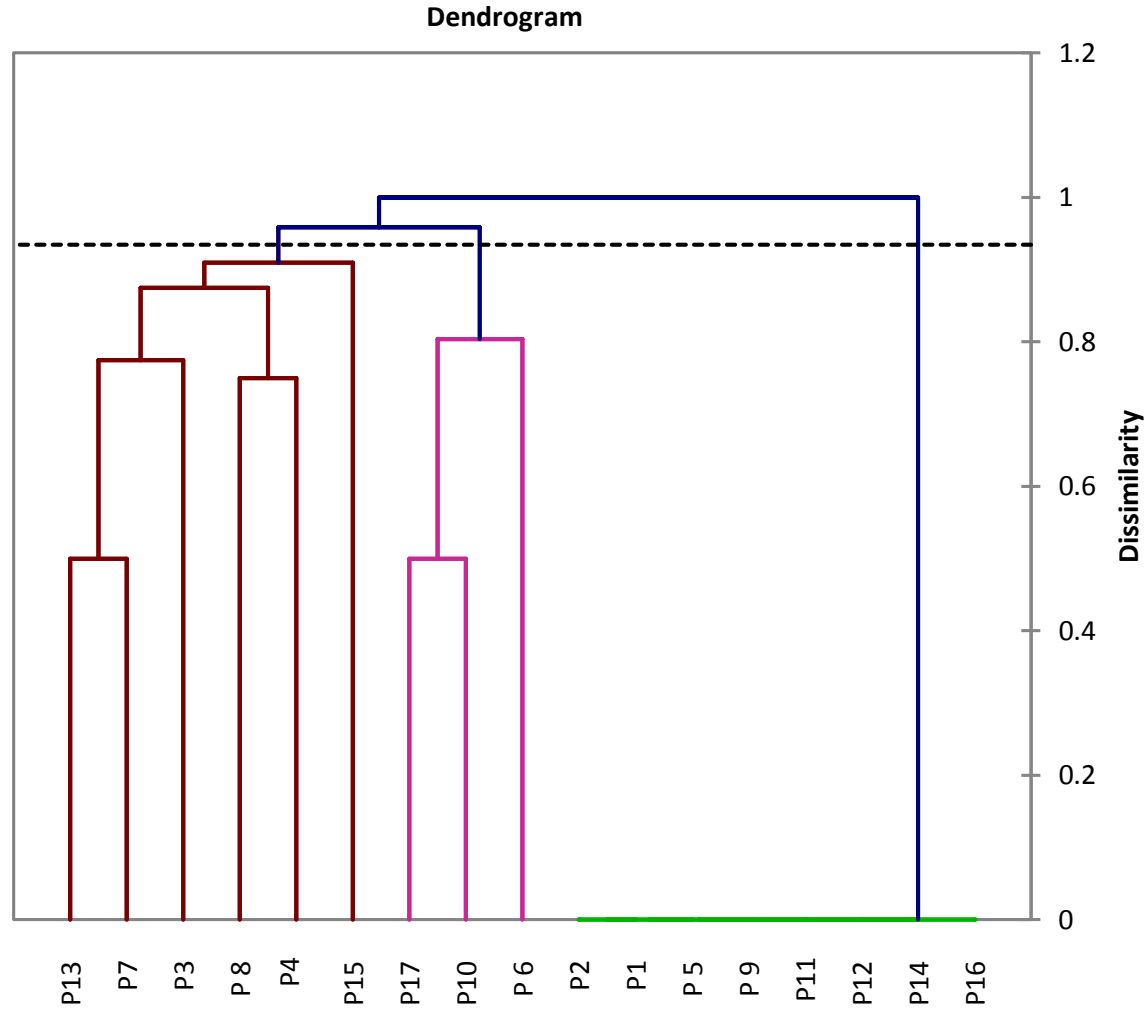

Figure 3. Clustering agglomerative complete linkage. 
three groups produced by the clustering analysis (Group I, Group II and Group III) (Figure 4).

Furthermore, the phytosociological analysis of the groups obtained with the previous multivariate analysis has allowed us to point out that the relives of $\mathrm{Abu}$ Salman Forest Reserve plots (P1, P2, P5, P9, P11, P12, P14, P16, P3, P4, P7, P8, $\mathrm{P} 13$ and P15) belong to the forest dominated by Pinus halepinsis Mill. recently described by Ighbareyeh J. M. H. et al. (2014) [19], this is a pine-tree forest characterized at the Idna village flora elements such as: Quercus libani G. Olivier, Quercus caliprinos Webb., Ceratonia siliqua L., Alain together with the endemic species Quercus look Kotschy, Tamarix palaestina Bertol and Tamarix jordanis L. this forest develops in the infra-thermomediterranean dry bioclimatic belt and it is growing in Mediterranean maquis and forest [2] [3], with the terra rose and brown rendzinas territory, we proposed pine-tree forest (Abu Salman Forest Reserve), group I or association ASL 1: 1 - 16 Pino halepinsis-Quercetum lookii (Table 1).

Groups II include species collected in Abu Salman Forest Reserve, a relives 3 to 15 represents the infra-thermotropical forest, and dry-subhumid ombrotype (Annual ombrothermic index $=1.5-2.8$ [2] [3], which is $3.5-6 \mathrm{~m}$ high, with a $45 \%$ - 90\% cover rate. This forest is characterized by the endemic trees Pyrus syriaca Boiss. Alain Pistacia palaestina Boiss., and growing in Mediterranean maquis and forest, therefore we proposed the association ASL 2 or Group II: 3 - 15 Pistacio paletinae-Ceratonietum siliquae (Table 2).

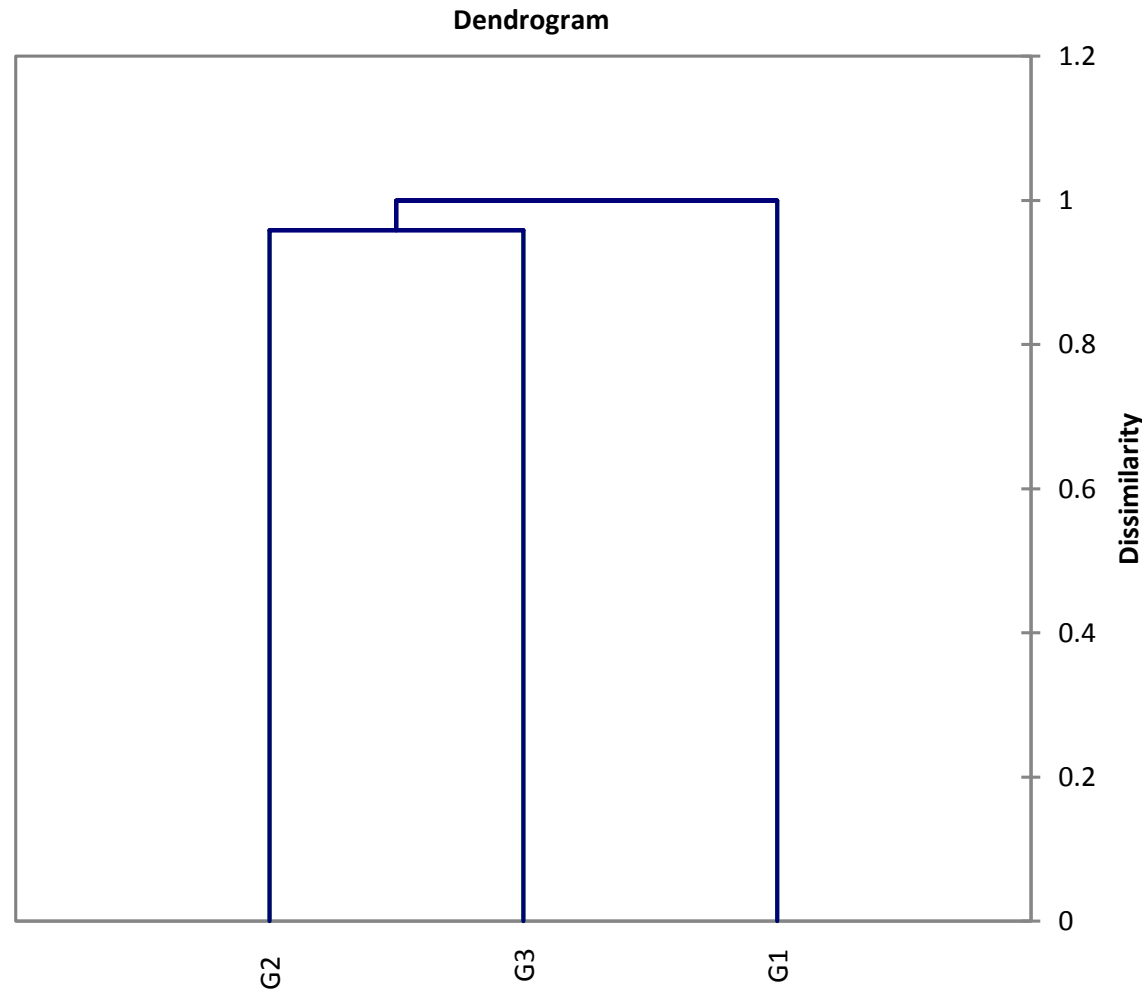

Figure 4. Cluster analysis for dry forest communities in the east south Idna-Hebron of Palestine. 
Table 1. Pino halepinsis-Quercetum lookii.

\begin{tabular}{|c|c|c|c|c|c|c|c|c|c|c|}
\hline Species & \multicolumn{10}{|c|}{$\mathbf{P}$} \\
\hline Altitude in meter & 533 & 497 & 522 & 540 & 611 & 488 & 555 & 470 & $\mathbf{R}$ & $S$ \\
\hline Surface in $\mathrm{m}^{2}$ & 100 & 120 & 285 & 280 & 250 & 300 & 270 & 280 & $\mathbf{E}$ & $\mathrm{T}$ \\
\hline Cover rate $\%$ & 70 & 35 & 45 & 60 & 90 & 85 & 60 & 45 & $s$ & A \\
\hline Average height of veg. (m) & 4 & 4.5 & 3.5 & 5 & 5.5 & 4 & 4 & 3.5 & $\mathbf{N}$ & $\mathrm{T}$ \\
\hline Slope \% & 10 & 5 & 15 & 5 & 5 & 10 & 5 & 5 & $\mathrm{C}$ & $\mathrm{U}$ \\
\hline Orientation & $\mathrm{W}$ & $\mathrm{w}$ & $\mathrm{w}$ & $\mathbf{N}$ & E & $\mathbf{E}$ & $\mathbf{N}$ & $\mathbf{E}$ & I & $S$ \\
\hline Number field relive & 1 & 2 & 3 & 4 & 21 & 22 & 23 & 25 & A & \\
\hline Grid number & P1 & P2 & P5 & P9 & P11 & $\mathrm{P} 12$ & $\mathrm{P} 14$ & P16 & $S$ & \\
\hline Order number & 1 & 2 & 3 & 4 & 5 & 6 & 7 & 8 & & \\
\hline \multicolumn{11}{|l|}{$\begin{array}{l}\text { Characteristic of the } \\
\text { association and upper units }\end{array}$} \\
\hline Quercus look Kotschy & 4 & 2 & 2 & 3 & 3 & 2 & 2 & 2 & $\mathrm{~V}$ & $\mathrm{E}$ \\
\hline Quercus libani G. Olivier & 3 & 2 & 1 & 1 & 2 & 2 & & & IV & $\mathrm{N}$ \\
\hline Pinus halepensis Mill & 2 & & 2 & & 1 & & 2 & 1 & III & $\mathrm{N}$ \\
\hline Quercus caliprinos Webb. & 2 & & 1 & & 2 & 2 & & 1 & III & $\mathrm{N}$ \\
\hline Ceratonia siliqua L. & & & 1 & & & 2 & & & II & $\mathrm{N}$ \\
\hline \multicolumn{11}{|l|}{ Companions } \\
\hline Rubus idaeus $\mathrm{L}$. & 1 & 2 & 1 & 1 & 2 & 2 & & & $\mathrm{~V}$ & $\mathrm{~N}$ \\
\hline Rubus fruticosus $\mathrm{L}$. & 2 & & 1 & 2 & 1 & & 2 & 1 & $\mathrm{~V}$ & $\mathrm{~N}$ \\
\hline Rhus tripartita Lobadium Raf. & & & 1 & 1 & 2 & 1 & & 2 & & III \\
\hline Rhus coriaria $\mathrm{L}$. & & & & 1 & 1 & & & 1 & I & $\mathrm{N}$ \\
\hline Tamarix palaestina Bertol & & & & 2 & & & 2 & & I & $\mathrm{E}$ \\
\hline Tamarix parviflora $D C$ & 1 & & & 2 & & & 2 & & I & $\mathrm{N}$ \\
\hline Tamarix tetragyna Ehrenb & 1 & & & & 1 & & 2 & 1 & II & $\mathrm{N}$ \\
\hline Tamarix jordanis $\mathrm{L}$. & & & & & 1 & & & 1 & I & $\mathrm{E}$ \\
\hline Pistacia palaestina Boiss. & & 1 & 2 & & & 2 & & & II & $\mathrm{E}$ \\
\hline Pistacia saportae Burnat & & & & & 1 & & & & I & $\mathrm{N}$ \\
\hline Pistacia lentiscus $\mathrm{L}$. & & & & 2 & & & & & I & $\mathrm{N}$ \\
\hline Pistacia atlantica Desf. & 2 & & & 2 & 1 & & & & II & $\mathrm{N}$ \\
\hline Pyrus syriaca Boiss. & & & & 1 & 1 & & & & I & $\mathrm{E}$ \\
\hline Quercus ithaburensi Kotschy & 2 & & 2 & & 1 & & & 1 & II & $\mathrm{N}$ \\
\hline Echinops philistaeus Feinbrun \& Zohary & 1 & & & 1 & 1 & & & & I & $\mathrm{E}$ \\
\hline Cupressus sempervirens $\mathrm{L}$. & & & & 1 & & & & & I & $\mathrm{N}$ \\
\hline Morus nigra $\mathrm{L}$. & & & & & 1 & & 2 & 1 & I & $\mathrm{N}$ \\
\hline Ficus carica $\mathrm{L}$. & & 1 & & 1 & & & & 1 & I & $\mathrm{N}$ \\
\hline Populus euphratica $\mathrm{L}$. & & & & 1 & 1 & & & & I & $\mathrm{N}$ \\
\hline Abies cilicica (Antoine \& Koschy) & & & & & & 1 & & & & I \\
\hline Salix acmophylla Boiss & & & & 2 & & & & 1 & I & $\mathrm{N}$ \\
\hline Fagus crenata Blume & & & & 1 & 1 & & 2 & & I & $\mathrm{E}$ \\
\hline Cercis siliquastrum $\mathrm{L}$. & & & & 2 & 1 & & 2 & & I & $\mathrm{N}$ \\
\hline Cercis siliquastrum $\mathrm{L}$. & & & & 2 & & & 2 & & I & $\mathrm{N}$ \\
\hline Cerasus microcarpa Boiss & & & & & 1 & & 2 & & I & $\mathrm{N}$ \\
\hline Rhamnus punctata Boiss & 2 & & & & & & & 1 & I & $\mathrm{N}$ \\
\hline Rhamnus lycioides Brot & 2 & & & 2 & 1 & & & 1 & II & $\mathrm{N}$ \\
\hline
\end{tabular}




\section{Continued}

\begin{tabular}{|c|c|c|c|c|c|c|c|c|}
\hline Rhamnus Palestaestina Boiss & 3 & & 2 & 1 & & 2 & II & $\mathrm{E}$ \\
\hline Arbutus andrachne $\mathrm{L}$. & & & 2 & & & 1 & I & $\mathrm{E}$ \\
\hline Olea europaea L. & & 1 & & & 2 & & I & $\mathrm{N}$ \\
\hline Pinus canariensis $\mathrm{L}$. & 1 & & & 1 & & 1 & I & $\mathrm{N}$ \\
\hline Pinus brutia $\mathrm{L}$. & 1 & & 1 & & & 1 & I & $\mathrm{N}$ \\
\hline Pinus pinea $\mathrm{L}$. & 1 & & & 1 & & 1 & I & $\mathrm{N}$ \\
\hline Cupressus sempervirens Var horizontalis & 1 & 1 & & & & 1 & I & $\mathrm{N}$ \\
\hline Cupressus arizonica $\mathrm{L}$. & & 1 & & & & & I & $\mathrm{N}$ \\
\hline Cupressus macrocarpaa $\mathrm{L}$. & & & & 1 & & 1 & I & $\mathrm{N}$ \\
\hline Crataegus aronia $\mathrm{L}$. & 1 & & 1 & & & & I & $\mathrm{N}$ \\
\hline Crataegus azarolus $\mathrm{L}$. & & & & & & 1 & I & $\mathrm{N}$ \\
\hline Styrax officinalis L. & 1 & & & 1 & & & I & $\mathrm{N}$ \\
\hline Lycium barbatum $\mathrm{L}$. & & & 1 & & & 1 & I & $\mathrm{N}$ \\
\hline Acer syriscum $\mathrm{L}$. & & 1 & & 1 & & & I & $\mathrm{E}$ \\
\hline Acacia cyanophylla L. & 1 & & 1 & & & & I & $\mathrm{N}$ \\
\hline Eucalyptus camaldulensis $\mathrm{L}$. & & & 1 & & & & & I \\
\hline Melia azedarach L. & 1 & & 1 & 1 & & & I & $\mathrm{N}$ \\
\hline
\end{tabular}

Table 2. Pistacio palestinae-Ceratonietum siliquae.

\begin{tabular}{|c|c|c|c|c|c|c|c|c|}
\hline Species & \multicolumn{8}{|c|}{$\mathbf{P}$} \\
\hline Altitude in meter & 450 & 466 & 490 & 545 & 460 & 488 & $\mathbf{R}$ & $S$ \\
\hline Surface in $\mathrm{m}^{2}$ & 270 & 120 & 270 & 300 & 250 & 300 & E & $\mathrm{T}$ \\
\hline Cover rate $\%$ & 90 & 35 & 75 & 60 & 90 & 85 & $S$ & A \\
\hline Average height of veg. (m) & 3.5 & 4 & 3.5 & 5 & 6 & 4 & $\mathrm{~N}$ & $\mathrm{~T}$ \\
\hline Slope \% & 5 & 5 & 15 & 10 & 5 & 10 & $\mathrm{C}$ & $\mathrm{U}$ \\
\hline Orientation & $\mathbf{E}$ & $\mathrm{E}$ & $\mathbf{E}$ & $\mathbf{N}$ & $\mathrm{E}$ & $\mathbf{E}$ & I & $S$ \\
\hline Number field relive & 27 & 28 & 29 & 30 & 32 & 33 & A & \\
\hline Grid number & P3 & P4 & P7 & P8 & P13 & P15 & $S$ & \\
\hline Order number & 1 & 2 & 3 & 4 & 5 & 6 & & \\
\hline \multicolumn{9}{|l|}{$\begin{array}{l}\text { Characteristic of the } \\
\text { association and upper units }\end{array}$} \\
\hline Ceratonia siliqua L. & 4 & 2 & 1 & 2 & 2 & 2 & $\mathrm{~V}$ & $\mathrm{~N}$ \\
\hline Pistacia lentiscus L. & 1 & & 1 & 2 & 3 & & III & $\mathrm{N}$ \\
\hline Pistacia palaestina Boiss. & 2 & 2 & 2 & 2 & 1 & 2 & IV & $\mathrm{E}$ \\
\hline Pinus halepensis Mill. & & 2 & 2 & 2 & 1 & & II & $\mathrm{N}$ \\
\hline Rubus fruticosus $\mathrm{L}$. & 2 & & 1 & 2 & 1 & & II & $\mathrm{N}$ \\
\hline Rhus tripartita Lobadium Raf. & & & & & 2 & 1 & & I \\
\hline Rhus coriaria $\mathrm{L}$. & & & & 1 & 1 & & I & $\mathrm{N}$ \\
\hline Quercus look Kotschy & & 2 & 2 & 1 & & 2 & II & $\mathrm{E}$ \\
\hline Quercus libani G. Olivier & 3 & & 1 & 1 & & 2 & II & $\mathrm{N}$ \\
\hline Quercus caliprinos Webb. & 2 & & 1 & & 2 & & I & $\mathrm{N}$ \\
\hline Pinus halepensis Mill. & 1 & 2 & & 1 & 2 & & I & $\mathrm{N}$ \\
\hline
\end{tabular}




\section{Continued}

\begin{tabular}{|c|c|c|c|c|c|c|c|c|}
\hline Companions & & & & & & & & \\
\hline Rubus idaeus $\mathrm{L}$. & + & 2 & & & 2 & & I & $\mathrm{N}$ \\
\hline Tamarix palaestina Bertol & 2 & & 2 & 2 & & & II & $\mathrm{E}$ \\
\hline Tamarix parviflora $D C$ & 1 & & & 2 & & & I & $\mathrm{N}$ \\
\hline Tamarix tetragyna Ehrenb & 1 & & & & 1 & & I & $\mathrm{N}$ \\
\hline Tamarix jordanis $\mathrm{L}$. & & & 1 & & 1 & & I & $\mathrm{E}$ \\
\hline Pistacia saportae Burnat & & & & & 1 & & I & $\mathrm{N}$ \\
\hline Pistacia lentiscus $\mathrm{L}$. & & & & 2 & & & I & $\mathrm{N}$ \\
\hline Pistacia atlantica Desf. & 2 & & & & 1 & & I & $\mathrm{N}$ \\
\hline Pyrus syriaca Boiss. & & & & 1 & 1 & & I & $\mathrm{E}$ \\
\hline Quercus ithaburensi Kotschy & 2 & & & 1 & & & I & $\mathrm{N}$ \\
\hline Echinops philistaeus Feinbrum \& Zohary & 1 & & & 1 & 1 & & I & $\mathrm{E}$ \\
\hline Cupressus sempervirens $\mathrm{L}$. & & & & 1 & & & I & $\mathrm{N}$ \\
\hline Morus nigra $\mathrm{L}$. & & & & & 1 & & I & $\mathrm{N}$ \\
\hline Ficus carica $\mathrm{L}$. & & & & 1 & & & I & $\mathrm{N}$ \\
\hline Populus euphratica $\mathrm{L}$. & & & & & 1 & & I & $\mathrm{N}$ \\
\hline Abies cilicica (Antoine \& Koschy) & & & & & & 1 & & I \\
\hline Salix acmophylla Boiss & & & & 2 & & & I & $\mathrm{N}$ \\
\hline Fagus crenata Blume & & & & & 1 & & I & $\mathrm{E}$ \\
\hline Cercis siliquastrum $\mathrm{L}$. & & & & 1 & 1 & & I & $\mathrm{N}$ \\
\hline Cerasus microcarpa Boiss & & & & & 1 & & I & $\mathrm{N}$ \\
\hline Quercus ithaburensi Kotschy & 2 & & & 2 & 1 & & I & $\mathrm{N}$ \\
\hline Rhamnus punctata Boiss & & & & & & & I & $\mathrm{N}$ \\
\hline Rhamnus lycioides Brot & & & & 2 & 1 & & I & $\mathrm{N}$ \\
\hline Rhamnus Palestaestina Boiss. & 3 & & & 1 & 1 & & II & $\mathrm{E}$ \\
\hline Arbutus andrachne $\mathrm{L}$. & & & & 1 & & & I & $\mathrm{E}$ \\
\hline Olea europaea L. & 1 & & & & & & I & $\mathrm{N}$ \\
\hline Pinus canariensis $\mathrm{L}$. & & & 1 & & 1 & 1 & I & $\mathrm{N}$ \\
\hline Pinus brutia $\mathrm{L}$. & & & & 1 & & & I & $\mathrm{N}$ \\
\hline Pinus pinea $\mathrm{L}$. & & & 1 & & 1 & 1 & I & $\mathrm{N}$ \\
\hline Cupressus sempervirens Var horizontalis & 1 & 1 & & & & & I & $\mathrm{N}$ \\
\hline Cupressus arizonica $\mathrm{L}$. & & & 1 & & & & I & $\mathrm{N}$ \\
\hline Cupressus macrocarpaa L. & & & & & & 1 & I & $\mathrm{N}$ \\
\hline Crataegus aronia $\mathrm{L}$. & 1 & & & 1 & & & I & $\mathrm{N}$ \\
\hline Crataegus azarolus $\mathrm{L}$. & & & & & & & I & $\mathrm{N}$ \\
\hline Styrax officinalis $\mathrm{L}$. & 1 & & & & & 1 & I & $\mathrm{N}$ \\
\hline Lycium barbatum $\mathrm{L}$. & & & & 1 & & & I & $\mathrm{N}$ \\
\hline Acer syriscum $\mathrm{L}$. & & & 1 & & & 1 & I & $\mathrm{E}$ \\
\hline Acacia cyanophylla L. & & & & 1 & 1 & & I & $\mathrm{N}$ \\
\hline Eucalyptus camaldulensis $\mathrm{L}$. & & & & 1 & & & & I \\
\hline Melia azedarach L. & & & & 1 & & 1 & I & $\mathrm{N}$ \\
\hline
\end{tabular}


Moreover, relives 6 to 17 including the Group III correspond to the forest of Pinus halepinus Mill.- Tamaricetum Palestineae. It is a tangled, plant forest 3.5 $6 \mathrm{~m}$ high, with a $40 \%-80 \%$ cover rate. This plants are characterized by Rubus idaeus L., Pinus halepensis Mill., Rhamnus punctata Boiss, Rhamnus lycioides Brot. \& Rhamnus Palestaestina Boiss. and the local endemic plant Rhamnus Palestaestina Boiss. Echinops philistaeus Feinbrun \& Zohary and Alain, we proposed Group III or association ASL3: 6 - 17 Quercus libanii-Tamaricetum Palestineae (Table 3).

In the other side, we include the forests growing in dry, sub-humid environments, it is probably necessary to create a new alliance to include all forests, and differences between the different associations proposed can be seen in the synthetic Table 4.

Finally, it is possible to say that Abu Salman Forest Reserve will be reduced to some plants or will be removed in the future due to the acceleration of construction (building houses and human activities), it is lies within the area classified B, this means that people can build houses and do their various activities, because it is located under Palestinian control, while the area $\mathrm{C}$ and $\mathrm{D}$ are located under Israeli control, don't it permits to construct of building houses and any activities.

Climate change [2] [3], the growth of the population, the increase in the population density, and the occupation of the Palestinian territories occupied in 1967, including Abu Salman Forest Reserve and other Palestinian areas, will give us a serious indication of the loss of many endemic, semi-endemic and native plants in Palestine, thus eliminating a large part of the vegetation, flora and biodiversity in the future of Palestine.

\section{Conclusions}

Our study reveal that the 17 grids analyzed are clearly divided into three different groups, all of these groups represent the potential natural vegetation and with a very little substitution scrublands. The floristic analysis revealed that $20.83 \%$ of these species are endemic especial in Abu Salman Forest Reserve. However, we indicated that the forests of Hebron as Idna village belong to different forests groups: Pine-tree forest as Pinus halepinus Mill., Arbutus andrachne L. and $\mathrm{Cu}$ pressus arizonica L., Mediterranean maquis and forest as Quercus look Kotschy, Pistacia palestina L., Carob-lentisk maquis as Ceratonia siliqua L. and Pistacia lentiscus L., Everegreen oak maquis as Quercus caliprinos Webb, Rhamnus Palestaestina Boiss. and Rhamnus punctata Boiss., Deciduous oak forests as Pistacia palestina Boiss. and Pistacia saportae Burnat., and Reprian forests as Tamarix palaestina Bertoland Tamarix Jordani.

Associations described as new:

Pino halepinsis-Quercetum lookii ${ }^{*}$.

Pistacio paletinae-Ceratonietum siliquae*.

Quercus libanii-Tamaricetum palestineae*.

In the near area of Idna Village, associations described by Ighbareyeh J. M. H. et al., 2014 [3] were followed: 
Table 3. Quercus libanii- Tamaricetum palestineae.

\begin{tabular}{|c|c|c|c|c|c|c|c|c|}
\hline Species & \multicolumn{8}{|c|}{$\mathbf{P}$} \\
\hline Altitude in meter & 460 & 480 & 522 & 540 & 600 & 450 & $\mathbf{R}$ & $S$ \\
\hline Surface in $\mathrm{m}^{2}$ & 100 & 100 & 280 & 270 & 250 & 300 & $\mathbf{E}$ & $\mathrm{T}$ \\
\hline Cover rate $\%$ & 80 & 40 & 50 & 60 & 90 & 85 & $S$ & A \\
\hline Average height of veg. (m) & 4 & 3.5 & 3.5 & 5 & 5.5 & 4 & $\mathbf{N}$ & $\mathrm{T}$ \\
\hline Slope \% & 5 & 5 & 10 & 5 & 5 & 10 & $\mathrm{C}$ & $\mathrm{U}$ \\
\hline Orientation & $\mathrm{W}$ & $\mathrm{W}$ & $\mathrm{W}$ & $\mathbf{N}$ & $\mathrm{E}$ & $\mathbf{E}$ & I & $S$ \\
\hline Number field relive & 40 & 41 & 42 & 44 & 47 & 48 & A & \\
\hline Grid number & P6 & P7 & P17 & P8 & P13 & P15 & $S$ & \\
\hline Order number & 1 & 2 & 3 & 4 & 5 & 7 & & \\
\hline \multicolumn{9}{|l|}{$\begin{array}{l}\text { Characteristic of the } \\
\text { association and upper units }\end{array}$} \\
\hline Tamarix palaestina Bertol & 4 & 2 & 2 & 1 & 2 & 2 & $\mathrm{~V}$ & $\mathrm{E}$ \\
\hline Tamarix parviflora $D C$ & 2 & 2 & 1 & 1 & 2 & 2 & IV & $\mathrm{N}$ \\
\hline Tamarix tetragyna Ehrenb & 1 & 2 & 2 & 1 & 2 & 2 & III & $\mathrm{N}$ \\
\hline $\begin{array}{l}\text { Tamarix jordanis } \mathrm{L} . \\
\text { Companions }\end{array}$ & & 1 & & & & & I & $\mathrm{E}$ \\
\hline Pinus halepensis Mill. & 2 & & 1 & 2 & 2 & & III & $\mathrm{N}$ \\
\hline Quercus look Kotschy & & & 1 & 1 & & & I & $\mathrm{E}$ \\
\hline Quercus libani G. Olivier & 2 & & 2 & 2 & 1 & 1 & IV & $\mathrm{N}$ \\
\hline Quercus caliprinos webb. & & & & & 1 & & I & $\mathrm{N}$ \\
\hline Ceratonia siliqua L. & & & 1 & & & 2 & II & $\mathrm{N}$ \\
\hline Rubus idaeus $\mathrm{L}$. & 1 & & & 1 & & 2 & I & $\mathrm{N}$ \\
\hline Rubus fruticosus $\mathrm{L}$. & 2 & & 1 & 2 & 1 & & III & $\mathrm{N}$ \\
\hline Rhus tripartita Lobadium Raf. & & & & & 2 & 1 & & I \\
\hline Rhus coriaria $\mathrm{L}$. & & & & 1 & 1 & & I & $\mathrm{N}$ \\
\hline Pistacia palaestina Boiss. & & & 2 & & & 2 & II & $\mathrm{E}$ \\
\hline Pistacia saportae Burnat & & & & & 1 & & I & $\mathrm{N}$ \\
\hline Pistacia lentiscus L. & & & & 2 & & & I & $\mathrm{N}$ \\
\hline Pistacia atlantica Desf. & 2 & & & 2 & 1 & & II & $\mathrm{N}$ \\
\hline Pyrus syriaca Boiss. & & & & 1 & 1 & & I & $\mathrm{E}$ \\
\hline Quercus ithaburensi Kotschy & 2 & & & 2 & 1 & & II & $\mathrm{N}$ \\
\hline Echinops philistaeus Feinbrun \& Zohary & 1 & & & 1 & 1 & & I & $\mathrm{E}$ \\
\hline Cupressus sempervirens $\mathrm{L}$. & & 1 & & & & & I & $\mathrm{E}$ \\
\hline Morus nigra L. & & & & & 1 & & I & $\mathrm{N}$ \\
\hline Ficus carica $\mathrm{L}$. & & 1 & & 1 & & & I & $\mathrm{N}$ \\
\hline Populus euphratica L. & & & & 1 & 1 & & I & $\mathrm{N}$ \\
\hline Abies cilicica (Antoine \& Koschy) & & & & & & 1 & & I \\
\hline Salix acmophylla Boiss & & & & 2 & & & I & $\mathrm{N}$ \\
\hline Fagus crenata Blume & & & 1 & & 1 & & I & $\mathrm{E}$ \\
\hline
\end{tabular}




\section{Continued}

\begin{tabular}{|c|c|c|c|c|c|c|c|c|}
\hline Cercis siliquastrum $\mathrm{L}$. & & & & 2 & 1 & & I & $\mathrm{N}$ \\
\hline Cerasus microcarpa Boiss & & & 1 & & 1 & & I & $\mathrm{N}$ \\
\hline Rhamnus punctata Boiss & 2 & & & & & & I & $\mathrm{N}$ \\
\hline Rhamnus lycioides Brot & & & & 2 & 1 & & I & $\mathrm{N}$ \\
\hline Rhamnus Palestaestina Boiss & 3 & & 2 & 2 & 1 & & III & $\mathrm{E}$ \\
\hline Arbutus andrachne $\mathrm{L}$. & & & & & & & I & $\mathrm{E}$ \\
\hline Olea europaea L. & & 1 & & & & & I & $\mathrm{N}$ \\
\hline Pinus canariensis $\mathrm{L}$. & & & 1 & & 1 & 1 & I & $\mathrm{N}$ \\
\hline Pinus brutia L. & & & & 1 & & & I & $\mathrm{N}$ \\
\hline Pinus pinea $\mathrm{L}$. & & & 1 & & 1 & 1 & I & $\mathrm{N}$ \\
\hline Cupressus sempervirens Var horizontalis & 1 & 1 & & & & & I & $\mathrm{N}$ \\
\hline Cupressus arizonica $\mathrm{L}$. & & & 1 & & & & I & $\mathrm{N}$ \\
\hline Cupressus macrocarpaa L. & & & & & & 1 & I & $\mathrm{N}$ \\
\hline Crataegus aronia L. & 1 & & & 1 & & & $\mathrm{I}$ & $\mathrm{N}$ \\
\hline Crataegus azarolus $\mathrm{L}$. & & & & & & & I & $\mathrm{N}$ \\
\hline Styrax officinalis L. & & & & & 1 & 1 & I & $\mathrm{N}$ \\
\hline Lycium barbatum L. & & 1 & & 1 & & & I & $\mathrm{N}$ \\
\hline Acer syriscum L. & & & 1 & & & 1 & I & $\mathrm{E}$ \\
\hline Acacia cyanophylla L. & & 1 & & & 1 & & I & $\mathrm{N}$ \\
\hline Eucalyptus camaldulensis $\mathrm{L}$. & & & & 1 & & & & I \\
\hline Melia azedarach L. & 1 & & & & & 1 & I & $\mathrm{N}$ \\
\hline
\end{tabular}

$\mathrm{N}$ : native and $\mathrm{E}$ : endemic.

Table 4. Synthetic representation ASL4.

\begin{tabular}{cccccccc}
\hline \multicolumn{7}{c}{ Synthetic representation ASL4 } & \\
\hline Species & ASL1 & ASL2 & ASL3 & Life Form & Status & Family \\
\hline Quercus look Kotschy & V & II & II & T & E & Fagaceae \\
Quercus libani G. Olivier & IV & III & I & T & N & Fagaceae \\
Quercus caliprinos webb. & III & I & I & T & N & Fagaceae \\
Quercus ithaburensi Kotschy & II & I & II & T & N & Fagaceae \\
Fagus crenata Blume & I & I & I & T & E & Fagaceae \\
Pinus halepinsis Mill. & III & II & III & T & N & Pinaceae \\
Pinus canariensis L. & I & I & I & T & N & Pinaceae \\
Pinus brutia L. & I & I & I & T & N & Pinaceae \\
Pinus pinea L. & I & I & I & T & N & Pinaceae \\
Abies cilicica (Antoine \& Koschy) & I & I & I & T & N & Pinaceae \\
Cupressus sempervirens L. & I & I & II & T & N & Cupressaceae \\
Sempervirens Var horizontalis Cupressus & I & I & I & T & N & Cupressaceae \\
Cupressus arizonica L. & I & I & I & T & N & Cupressaceae \\
\hline
\end{tabular}




\section{Continued}

\begin{tabular}{|c|c|c|c|c|c|c|}
\hline Cupressus macrocarpaa L. & I & I & I & $\mathrm{T}$ & $\mathrm{N}$ & Cupressaceae \\
\hline Ceratonia siliqua L. & $\mathrm{V}$ & II & II & $\mathrm{T}$ & $\mathrm{N}$ & Fabaceae \\
\hline Cercis siliquastrum $\mathrm{L}$. & I & I & I & $\mathrm{T}$ & $\mathrm{N}$ & Fabaceae \\
\hline Rubus idaeus $\mathrm{L}$. & II & I & I & $\mathrm{T}$ & $\mathrm{N}$ & Rosaceae \\
\hline Rubus fruticosus $\mathrm{L}$. & $\mathrm{V}$ & II & III & $\mathrm{T}$ & $\mathrm{N}$ & Rosaceae \\
\hline Cerasus microcarpa Boiss & I & I & I & $\mathrm{Sh}$ & $\mathrm{N}$ & Rosaceae \\
\hline Crataegus aronia $\mathrm{L}$. & I & I & I & $\mathrm{T}$ & $\mathrm{N}$ & Rosaceae \\
\hline Crataegus azarolus $\mathrm{L}$. & I & I & I & $\mathrm{T}$ & $\mathrm{N}$ & Rosaceae \\
\hline Pyrus syriaca Boiss. & I & I & I & $\mathrm{T}$ & $\mathrm{E}$ & Rosaceae \\
\hline Tamarix palaestina Bertol & I & II & $\mathrm{V}$ & $\mathrm{T}$ & $\mathrm{E}$ & Tamaricaceae \\
\hline Tamarix parviflora $D C$ & I & I & IV & $\mathrm{T}$ & $\mathrm{N}$ & Tamaricaceae \\
\hline Tamarix tetragyna Ehrenb. & II & I & III & $\mathrm{T}$ & $\mathrm{N}$ & Tamaricaceae \\
\hline Tamarix jordanis L. & I & I & I & $\mathrm{T}$ & $\mathrm{E}$ & Tamaricaceae \\
\hline Pistacia palaestina Boiss. & II & IV & II & $\mathrm{T}$ & $\mathrm{E}$ & Anacardiaceae \\
\hline Pistacia saportae Burnat & I & I & I & $\mathrm{T}$ & $\mathrm{N}$ & Anacardiaceae \\
\hline Pistacia lentiscus L. & I & I & I & $\mathrm{T}$ & $\mathrm{N}$ & Anacardiaceae \\
\hline Pistacia atlantica Desf. & II & I & II & $\mathrm{T}$ & $\mathrm{N}$ & Anacardiaceae \\
\hline Rhus tripartita Lobadium Raf. & III & I & I & $\mathrm{T}$ & $\mathrm{N}$ & Anacardiaceae \\
\hline Rhus coriaria $\mathrm{L}$. & I & I & I & $\mathrm{T}$ & $\mathrm{N}$ & Anacardiaceae \\
\hline Morus nigra L. & I & I & I & $\mathrm{T}$ & $\mathrm{N}$ & Moraceae \\
\hline Ficus carica L. & I & I & I & $\mathrm{T}$ & $\mathrm{N}$ & Moraceae \\
\hline Populus euphratica L. & I & I & I & $\mathrm{T}$ & $\mathrm{N}$ & Salicaceae \\
\hline Salix acmophylla Boiss & I & I & I & $\mathrm{T}$ & $\mathrm{N}$ & Salicaceae \\
\hline Rhamnus punctata Boiss & I & I & I & $\mathrm{T}$ & $\mathrm{N}$ & Rhamnaceae \\
\hline Rhamnus lycioides Brot & II & I & I & $\mathrm{T}$ & $\mathrm{N}$ & Rhamnaceae \\
\hline Rhamnus Palestaestina Boiss. & II & III & II & $\mathrm{T}$ & $\mathrm{E}$ & Rhamnaceae \\
\hline Arbutus andrachne $\mathrm{L}$. & I & I & I & Ar & $\mathrm{E}$ & Ericaceae \\
\hline Styrax officinalis L. & I & I & I & $\mathrm{T}$ & $\mathrm{N}$ & Styracaceae \\
\hline Lycium barbatum $\mathrm{L}$. & I & I & I & $\mathrm{T}$ & $\mathrm{N}$ & Solanaceae \\
\hline Acer syriscum $\mathrm{L}$. & I & I & I & $\mathrm{T}$ & $\mathrm{E}$ & Aceraceae \\
\hline Acacia cyanophylla L. & I & I & I & $\mathrm{T}$ & $\mathrm{N}$ & Mimosaceae \\
\hline Eucalyptus camaldulensis L. & I & I & I & $\mathrm{T}$ & $\mathrm{N}$ & Myrtaceae \\
\hline Melia azedarach L. & I & I & I & $\mathrm{T}$ & $\mathrm{N}$ & Meliaceae \\
\hline Olea europaea L. & I & I & I & $\mathrm{T}$ & $\mathrm{N}$ & Oleaceae \\
\hline Echinops philistaeus Feinbrun \& Zohary & I & I & I & Annual & $\mathrm{E}$ & Boraginaceae \\
\hline
\end{tabular}

Percentage of plant species presence in the samples and communities: $\mathrm{V}=100 \%, \mathrm{IV}=60.1 \%-80 \%$, III $=$ $40.1 \%-60 \%, \mathrm{II}=20.1 \%-40 \%$ and $\mathrm{I}=0.1 \%-20 \%$. N: Native and E: Endemic. Association (ASL), GI: Group one, G II: Group two, GIII: Group three, GI or ASL1: Pinus halepinus-Quercetum lookii., G2 or ASL2: Pistacio paletinae-Ceratonietum siliquae and G3 or ASL3: Quercus libanii-Tamaricetum palestineae. 
Pistacio palaestinae-Quercetum lokii*.

Capparido sinaicae-Ceratonietum siliquae.

Cerasus microcarpae-Quercetum ithaburensis* .

Pyro siriacae-Abietetum cilicicae*.

Abio ciliciae-Ceratonietum siliquae.

Periploco aphylli-Pinetum halepensis.

Cytisopsis pseudocytiso-Tamaricetum tetragynae.

Crataego sinaicae-Tamaricetum jordanii.

${ }^{*}$ Associations in which olive cultivation is possible.

\section{References}

[1] Ighbareyeh, J.M.H., Cano-Ortiz, A. and Cano, E. (2014) Biological and Bioclimatic Basis to Optimize Plant Production: Increased Economic Areas of Palestine. Agricultural Science Research Journal, 4, 10-20.

[2] Ighbareyeh, J.M.H., Cano-Ortiz, A. and Cano, E. (2014) Case Study: Analysis of the Physical Factors of Palestinian Bioclimate. American Journal of Climate Change, 3, 223-231. https://doi.org/10.4236/ajcc.2014.32021

[3] Ighbareyeh, J.M.H., Cano-Ortiz, A., Suliemieh, A.A.A., Ighbareyeh, M.M.H. and Cano, E. (2014) Phytosociology with Other Characteristic Biologically and Ecologically of Plant in Palestine. American Journal of Plant Sciences, 5, 3104-3118. https://doi.org/10.4236/ajps.2014.520327

[4] Ighbareyeh, J.M.H., Cano-Ortiz, A., Suliemieh, A.A.A., Ighbareyeh, M.M.H. and Cano, E. (2015) Assessing Crop Yield Sustainability under the Climatic and Bioclimatic Change in the Area of Palestine. American Journal of Climate Change, 4, 48-56. https://doi.org/10.4236/ajcc.2015.41005

[5] Cano-Ortiz, A. Ighbareyeh, J.M.H. and Cano, E. (2014) Bioclimatic Applications and Soil Indicators for Olive Cultivation (South of the Iberian Peninsula). Global Advanced Research Journal of Agricultural Science, 3, 433-438.

[6] Ighbareyeh, J.M.H., Cano-Ortiz, A., Suliemieh, A.A.A., Ighbareyeh, M.M.H. and Cano, E. (2015) Study of Biology and Bioclimatology of Date Palm (Phoenix dactylifera L.) to Optimize Yield and Increase Economic in Jericho and Gaza Cities of Palestine. International Journal of Research Studies in Biosciences, 3, 1-8.

[7] Ighbareyeh, J.M.H., Cano-Ortiz, A., Suliemieh, A.A.A., Ighbareyeh, M.M.H. and Cano, E. (2015) Assessment of Biology and Bioclimatology of Plant to Increase Economic in Palestine. International Journal of Research Studies in Biosciences, 3, $1-8$.

[8] Ighbareyeh, J.M.H., Cano-Ortiz, A., Suliemieh, A.A.A., Ighbareyeh, M.M.H. and Cano, E. (2015) Biology and Bioclimatology Applied on Plant in Palestine. International Journal of Research Studies in Biosciences, 3, 79-86.

[9] Ighbareyeh, J.M.H., Cano-Ortiz, A., Suliemieh, A.A.A., Ighbareyeh, M.M.H. and Cano, E. (2015) Study Effect of Biology and Bioclimatology Applied on Plant in the Area of Hebron at the South of Palestine. International Journal of Research Studies in Biosciences, 3, 56-64.

[10] Ighbareyeh, J.M.H., Cano-Ortiz, A., Suliemieh, A.A.A., Ighbareyeh, M.M.H. and Cano, E. (2015) Effect of Biology and Bioclimatology Applied on Plant in the Area of Jenin at the North of Palestine. International Journal of Research Studies in Bioscience, 3, 1-6. 
[11] Ighbareyeh, J.M.H., Cano-Ortiz, A., Suliemieh, A.A.A., Ighbareyeh, M.M.H. and Cano, E. (2015) Study of Climatology and Bioclimatology Applied on Plant in Area of Hebron in Palestine. International Multidisciplinary Research Journal, 5.

[12] Ighbareyeh, J.M.H., Cano-Ortiz, A., Cano Carmona, E., Ighbareyeh, M.M.H. and Suliemieh, A.A.A. (2015) Modeling of Biology and Bioclimatology Applied on Plant in Palestine. Swift Journal of Agricultural Research, 1, 21-27.

[13] Ighbareyeh, J.M.H., Cano-Ortiz, A., Suliemieh, A.A.A., Ighbareyeh, M.M.H. and Cano, E. (2015) Effect of Biology and Bioclimatology Applied Studies on Plant in the Area of Jerusalem in Palestine. International Journal of Research Studies in Biosciences, 3, 135-140.

[14] Ighbareyeh, J.M.H., Cano-Ortiz A., Cano, E., Ighbareyeh, M.M.H. and Suliemieh, A.A.A. (2016) Effect of Biology, Climatic and Bioclimatic Applied Studies on Plant: To Increase the Economy and Maintaining Food Security in the Jerusalem Occupied of Palestine. International Journal of Research Studies in Biosciences, 4, 54-60.

[15] Ighbareyeh, J.M.H., Cano-Ortiz, A., Suliemieh, A.A.A., Ighbareyeh, M.M.H. and Cano, E. (2016) Study of Biology and Bioclimatology Applied of Apricot (Prunus armeniaca L.): To Increase the Economy and Maintaining Food Security in Palestine. International Journal of Research Studies in Biosciences, 4, 12-20.

[16] Ighbareyeh, J.M.H., Cano-Ortiz, A., Suliemieh, A.A.A., Ighbareyeh, M.M.H. and Cano, E. (2016) Modeling of Biology and Bioclimatology Applied Studies on Plant in Palestine. International Journal of Development Research, 6, 9585-9590. http://www.journalijdr.com

[17] Ighbareyeh, J.M.H., Cano-Ortiz, A., Suliemieh, A.A.A., Ighbareyeh, M.M.H., Cano, E. and Hijjeh, S. (2016) Effect of Bioclimate Factors on Olive (Olea europea L.) Yield: To Increase the Economy and Maintaining Food Security in Palestine. International Journal of Development Research, 6, 10648-10652. http://www.journalijdr.com

[18] Ighbareyeh, J.M.H., Cano-Ortiz, A., Cano Carmona, E., Ighbareyeh, M.M.H., Suliemieh, A.A.A. and Hijjeh, S. (2017) Impact of Bioclimate and Climate Factors on Plant Yield in the Area of Jenin at the North of Palestine. International Journal of Current Research, 9, 44529-44535.

[19] Ighbareyeh, J.M.H., Cano-Ortiz, A., Cano Carmona, E., Suliemieh, A.A.A. and Ighbareyeh, M.M.H. (2017) Flora Endemic Rare and Bioclimate of Palestine. Open Access Library Journal, 4, e3977. https://doi.org/10.4236/oalib.1103977

[20] Ighbareyeh, J.M.H. and Carmona, E.C. (2017) Impact of Climate and Bioclimate Factors on Apricot (Prunus armeniaca L.) Yield to Increase Economy and Achieve Maintaining Food Security of Palestine. Open Access Library Journal, 4, e4119. https://doi.org/10.4236/oalib.1104119

[21] Ali-Shtayeh, M.S. and Jamous, R.M. (2002) Red List of Threatened Plants of the West Bank and Gaza Strip and the Role of Botanic Gardens in Their Conservation. Biodiversity and Environmental Sciences Studies Series, 2, 1-46.

[22] Danin, A. (2004) Distribution Altas of Plants in Flora Palaestina Area. 2nd Edition, Academy of Science and Humanities, Jerusalem, 520.

[23] Sawalha, K. (2005) Plant Biodiversity. Al-Quds University, Abu Dis.

[24] Zohary, M. (1962) Plant Life of Palestine. Ronald Press Company, New York.

[25] Braun-Blanquet, J. (1979) Fitosociología. Bases para el estudio de las comunidades vegetales. Blume, Madrid, $820 \mathrm{p}$.

[26] Pott, R. (2011) Phytosociology: A Modern Geobotanical Method. Plant Biosystems, 145, 9-18. https://doi.org/10.1080/11263504.2011.602740 
[27] Biondi, E. (2011) Phytosociology Today: Methodological and Conceptual Evolution. Plant Biosystems, 145, 19-29. https://doi.org/10.1080/11263504.2011.602748

[28] Van Der Maarel, E. (1979) Transformation of Cover-Abundance Values in Phytosociology and Its Effects on Community Similarity. Vegetatio, 39, 97-114. https://doi.org/10.1007/BF00052021

[29] Rivas-Martınez, S., Rivas Saenz, S. and Penas, A. (2011) Worldwide Bioclimatic Classification System. Global Geobot, 1, 1-634. 